BULLETIN Bulletin hispanique

HISPANIQUE Université Michel de Montaigne Bordeaux

$117-2 \mid 2015$

Métamorphose(s) : représentations et réécritures

Isabelle Tauzin, L'Amérique latine écartelée : pouvoir et violence à l'épreuve de la fiction (Lituma en los Andes, Abril rojo, Trabajos del reino)

Presses Universitaires de France, Collection CNED, Série Espagnol, 2012

Raphaël Estève

\title{
OpenEdition
}

Journals

Édition électronique

URL : http://journals.openedition.org/bulletinhispanique/4043

DOI : 10.4000/bulletinhispanique.4043

ISSN : $1775-3821$

Éditeur

Presses universitaires de Bordeaux

Édition imprimée

Date de publication : 15 décembre 2015

Pagination : 759-762

ISBN : 979-10-300-0041-2

ISSN : 0007-4640

Référence électronique

Raphaël Estève, «Isabelle Tauzin, L'Amérique latine écartelée : pouvoir et violence à l'épreuve de la fiction (Lituma en los Andes, Abril rojo, Trabajos del reino) », Bulletin hispanique [En ligne], 117-2 | 2015, mis en ligne le 15 décembre 2015, consulté le 23 septembre 2020. URL : http://journals.openedition.org/ bulletinhispanique/4043; DOI : https://doi.org/10.4000/bulletinhispanique.4043

Ce document a été généré automatiquement le 23 septembre 2020.

Tous droits réservés 


\section{Isabelle Tauzin, L'Amérique latine écartelée : pouvoir et violence à l'épreuve de la fiction (Lituma en los Andes, Abril rojo, Trabajos del reino)}

Presses Universitaires de France, Collection CNED, Série Espagnol, 2012

\section{Raphaël Estève}

\section{RÉFÉRENCE}

Isabelle Tauzin, L'Amérique latine écartelée : pouvoir et violence à l'épreuve de la fiction (Lituma en los Andes, Abril rojo, Trabajos del reino), Presses Universitaires de France, Collection CNED, Série Espagnol, 2012, 142 p.

1 L'ouvrage, consacré à trois romans au programme des sessions 2013 et 2014 de l'agrégation externe d'espagnol, se présente dès son entame comme un «manuel universitaire » permettant de « découvrir » l'œuvre de romanciers concernés par « une littérature indépendante et engagée ». Au-delà de cette découverte, c'est un véritable éclairage, beaucoup plus dense que l'extension du volume (142 pages) ne le laisse supposer, qu'Isabelle Tauzin nous propose ici des romans des Péruviens Vargas Llosa et Santiago Roncagliolo, et du Mexicain Yuri Herrera. Cet éclairage est particulièrement riche, puisque les clefs livrées par l'auteure sont aussi bien textuelles que culturelles ou contextuelles.

2 Après une introduction synthétisant quelques apports conceptuels quant à la nature intrinsèque du pouvoir et son évolution vers une fragmentation rendant caduque à la fois la forme politique explicite de la tyrannie d'un seul et le corrélat littéraire - les romans de la dictature - qui l'a accompagnée/dénoncée au $\mathrm{XX}^{\mathrm{e}}$ siècle en Amérique latine, la place est laissée à une étude spécifique et séparée des œuvres, à propos desquelles l'auteure précise que son approche des deux premières sera fondée « sur une 
connaissance objective et empathique du Pérou », dont Isabelle Tauzin est en effet une spécialiste reconnue.

3 La première partie du travail est donc consacrée au roman de Mario Vargas Llosa, Lituma en los Andes, paru en 1993. Vargas Llosa n'étant pas uniquement un écrivain mondialement reconnu et nobélisé, mais également un acteur majeur sur la scène publique péruvienne, la présentation du contexte politique des années précédant immédiatement sa publication, où l'auteur est battu à l'élection présidentielle de 1990, est cruciale au moment de faire de son Lituma en los Andes une " arme de revanche politique ». Un découpage minutieux des séquences du roman est proposé sous forme de tableau : il est complété par une caractérisation très informative de Lituma, figure récurrente chez l'auteur. Sont également synthétisés les traits fondamentaux de l'écriture de Vargas Llosa, à qui la parole est sporadiquement laissée, puisqu'il a luimême théorisé un certain nombre de ces traits dans le cadre de son activité de critique littéraire: mentionnons le principe de récursivité, tel qu'il peut être symbolisé par l'image des poupées russes, ou encore le complémentaire de cette récursivité inclusive, le mécanisme des «vases communicants " (croisement des fils narratifs). Eu égard à la fonction exercée par le personnage titre, Lituma (membre des forces de l'ordre), des considérations très instructives sur le genre policier sont également reprises ici. Mais on retiendra avant tout deux dimensions de cette étude. Premièrement, l'attention soutenue prêtée au langage, qu'il s'agisse de l'éclairage onomastique (notamment à partir du quechua) qui est proposé des patronymes et surnoms les plus significatifs, des modalités de désignation des référents, ou de l'analyse des registres discursifs. Deuxièmement, l'éclairage des mises en regard - des superpositions - culturelles latentes dans le roman : et en premier lieu, la mise en lumière de son syncrétisme entre la mythologie du monde grec et celle du monde andin. L'analyse proposée observe donc scrupuleusement le positionnement anthropologique (faisant signe en permanence vers le hors-texte) du roman. Et ce, sans forcément adhérer à l'idéologie se dégageant du texte de Vargas Llosa. On louera à ce propos la ferme objectivité d'Isabelle Tauzin : elle relaie les critiques, idéologiques donc, proférées à l'endroit de l'auteur en les relativisant systématiquement par une re-contextualisation historique, culturelle ou linguistique qui enrichit et complexifie de façon adéquatement érudite les simplifications dont certaines querelles peuvent être le fruit.

4 En rupture avec le contournement nominal qui rend, d'une certaine façon, tangentielle l'approche du Sentier Lumineux dans Lituma en los Andes, mais en ayant toutefois intégré l'hypotexte que constitue ce dernier, on trouve le roman de Santiago Roncagliolo, Abril rojo, publié en 2006. C'est à lui qu'est concédée la place la plus importante dans l'étude d'Isabelle Tauzin (70 pages).

5 Après avoir rappelé l'influence de la pop culture sur Roncagliolo (les comics, le cinéma de Fincher, etc.), l'auteure va poursuivre son travail d'élucidation des références, directes ou implicites, indispensables à la bonne compréhension des enjeux du texte. Des éléments de contextualisation historique à la fois précis et synthétiques sont livrés à propos de la ville d'Ayacucho et surtout du groupe insurrectionnel du Sentier Lumineux, ainsi que de ses leaders intellectuels ayant directement inspiré certains personnages du roman. Ce point est d'importance car le travail linguistique et onomastique appliqué à Lituma en los Andes est dans le cas de Abril rojo enrichi d'une étude terminologique analysant parfaitement la rhétorique et le sous-texte d'un discours caractéristiquement idéologique ( «tics» conceptuels, propagande, mais aussi 
langue de bois de la part des autorités officielles). Une grande attention est ainsi concédée à l'explicitation des modalités linguistiques de la domination et de la réduction de l'altérité. De façon plus exclusivement littéraire, un important travail de discrimination stylistique, et donc idiolectale, des voix est également réalisé, ce qui éclaire considérablement l'enchevêtrement des points de vue à l'œuvre dans le roman de Roncagliolo. L'érudition opportune d'Isabelle Tauzin reconduit la dualité que nous avons déjà évoquée. Sont ainsi décodés et mis en dialogue, d'une part, les référents ou les mythes en rapport avec la culture andine locale, et d'autre part, des éléments de culture générale (" universels " ou "occidentaux», selon le point de vue auquel on souscrit), tels que les mythes d'ÆEdipe ou de Faust, ou bien sûr des classiques de la littérature comme E. A. Poe. Ces deux versants se rejoignent d'une certaine façon dans l'éclairage d'un sous-texte littéraire qui fait évoquer à plusieurs reprises la figure majeure de José María Arguedas et des textes indigénistes, dont Abril rojo reprend en les modernisant certains paradigmes (le « tinterillo » par exemple).

6 L'auteure accorde une grande importance à l'inscription dans le réel de l'écriture de Roncagliolo, "journaliste avant d'être écrivain », du fait notamment des témoignages authentiques incorporés dans le cadre de la fiction. Il y a, à partir de là, une ligne de démarcation (ou polarisation) idéologique autour de laquelle Isabelle Tauzin articule son propos : nous pouvons en distinguer ici deux modalités, qui reconduisent la dualité entre l'universel (supposé) et le particulier évoquée plus haut. La première, relevant de ce particulier, serait à présenter en termes de "décidabilité» de la violence, omniprésente dans le roman, et analysée en profondeur par l'étude. On la présentera comme une thèse endémique, à la fois officialisée hors-fiction par la commission d'enquête sous l'autorité de Mario Vargas Llosa, et reprise dans le texte par le personnage du commandant Carrión : c'est la barbarie - son «inculture » - indienne qui serait génératrice de violence. Il s'agit d'une thèse qu'Isabelle Tauzin s'emploie à invalider. La seconde modalité relève précisément de l'universel supposé, qui tente de résorber le particulier. C'est ici que prend tout son sens l'analyse très précise par l'auteure de l'intertexte politique du discours du Sentier Lumineux, qui renvoie on le sait au maoïsme. Car, si «tous les noms de guerre des cadres du Sentier Lumineux disent le refus de l'identité indienne, le choix contradictoire de l'identité créole », ce n'est bien entendu pas cette terminologie, qui oppose un particulier à un autre (indien vs créole), qui serait assumée par le marxisme-léninisme du Sentier Lumineux (PCP$\mathrm{SL}$ ) : il opposerait bien plutôt le particulier andin à l'universel abstrait sous les auspices duquel se place son rationalisme revendiqué. On notera par conséquent la mention pertinente du sentiérologue C. I. Degregori, qui caractérise le mouvement insurrectionnel par "un exceso de razón ». La reformulation de ce dernier en "culto divino a la razón " nous permet d'ailleurs de parfaitement comprendre l'appropriation de la symbolique chrétienne à partir de 1979 par le Sentier Lumineux : elle aurait vocation/prétention universalisante, à l'image (non-assumée par le PCP-SL) de la volonté d'évangélisation du Nouveau-Monde dès la découverte de celui-ci. Isabelle Tauzin note d'ailleurs que « la superposition de la symbolique religieuse et du discours extrémiste du Sentier Lumineux est une des singularités d'Abril rojo ", et en même temps ce qui a pu offrir une prise à la critique.

7 Le traitement du troisième roman, Trabajos del reino, publié en 2010 par Yuri Herrera, est plus synthétique, peut-être du fait de son inscription géographique et contextuelle qui le distingue des deux précédents: le Mexique du narcotrafic. Isabelle Tauzin témoigne pourtant d'une bonne connaissance de la réalité mexicaine, en complément 
de laquelle elle convoque de façon récurrente, et toujours avec à-propos, l'autorité éclairée de Carlos Monsiváis. On retrouve à l'œuvre dans cette dernière étude le double éclairage culturel qui fait la force du travail. Le Poème d'Hésiode ou les éléments traditionnels de la symbolique du corps du roi, opportunément analysés dans un roman naissant, entre autre, de l'intérêt de son auteur pour " la figura del artista de la corte », sont ainsi des clés de lecture faisant pendant à l'évocation locale de ces corridos, encore particularisables en narcocorridos, qui sont des formes de chants populaires, en l'occurrence disséminées dans le récit, et à la gloire du raccourci vers la réussite que constitue le narcotrafic. Point aveugle de cette "réussite", les exactions qui lui sont indispensables appellent chez l'auteure une appréhension à la fois théorique et empathique de la violence : l'horreur permise par la réification totale de l'humanité des victimes. On notera une fois encore le positionnement toujours remarquablement nuancé - parce qu'informé - de la part de l'auteure à propos des critiques formulées à l'encontre du texte analysé.

8 On dira en résumé que l'ouvrage s'acquitte parfaitement de la tâche qu'il s'était assignée : livrer les plus importantes clés de lecture des trois romans étudiés. Mais, bien au-delà de cet objectif, le travail d'Isabelle Tauzin offre au lecteur un bagage tout à fait conséquent pour appréhender méthodiquement toute œuvre littéraire relevant de cette aire culturelle qu'est l'Amérique latine.

\section{AUTEURS}

\section{RAPHAËL ESTÈVE}

Université Bordeaux Montaigne 